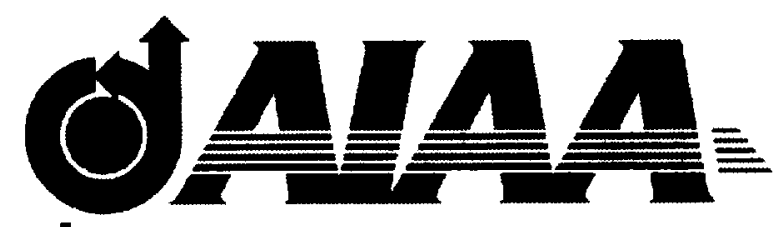

\title{
AIAA-2002-1654
}

\section{Random Walk Method for Potential Problems}

T. Krishnamurthy

NASA Langley Research Center, Hampton, VA 23681

I. S. Raju

NASA Langley Research Center, Hampton, VA 23681

\section{3 $^{\text {rd }}$ AIAA/ASME/ASCE/AHS/ASC Structures, Structural Dynamics, and Materials Conference 22-25 April, 2002 Denver, Colorado}

For permission to copy or republish, contact the American Institute of Aeronautics and Astronautics 1801 Alexander Bell Drive, Suite 500, Reston. VA 22091 



\title{
Random Walk Method for Potential Problems
}

\author{
T. Krishnamurthy \\ And \\ I. S. Raju ${ }^{\dagger}$ \\ NASA Langley Research Center, Hampton, VA 23681, U.S.A.
}

\begin{abstract}
A local Random Walk Method (RWM) for potential problems governed by Lapalace's and Poisson's equations is developed for two- and threedimensional problems. The RWM is implemented and demonstrated in a multiprocessor parallel environment on a Beowulf cluster of computers. A speed gain of 16 is achieved as the number of processors is increased from 1 to 23 .
\end{abstract}

\section{Introduction}

The Finite Element Method (FEM) is a widely used numerical method for structural analysis. For largescale structural analysis, FEM needs extensive preprocessing and hence may be costly and time consuming. The Boundary Element Method (BEM) is considered to be an alternative to FEM for a certain class of problems ${ }^{1,2}$. In the BEM, it is necessary to model only the boundary, and hence BEM reduces the dimensions of the problem. Both FEM and BEM have their own advantages and disadvantages. Hence methods to couple FEM and BEM have evolved ${ }^{3}$ to take advantages of both methods. A third class of methods denoted as meshless methods ${ }^{46}$ has been developed as a replacement for FEM and BEM. All the above-mentioned methods can be grouped as global methods. These global methods can provide solutions to stress, displacement, and other responses for all the points in the structure. The global methods also invariably need to form and invert a very large system matrix to obtain the complete field solution. Recently local methods such as the Random Walk Method (RWM) were proposed in reference 7 to obtain the solution at an arbitrary point, without having to obtain the complete field solution. These local methods are based on probabilistic interpretations of certain partial differential equations. For these local methods, there is no necessity to discretize the domain or the boundary. Also they are highly economical, if the solution is needed at only at a few selected points in the structure. These methods are simple to program and inherently parallel. This feature of the local methods makes it most suited for analysis in a cluster of computers, such as Beowulf cluster ${ }^{8}$. In the cluster, several computers processors (CPU) are networked together such that each processor uses its own local memory but is able to communicate with other processors by sending and receiving messages. In the present paper the Random Walk Method (RWM) for potential problems (governed by Laplace and Poisson's equations) for two- and three-dimensions is adopted from reference 7 and implemented in a Beowulf cluster of computers. The efficiency of the RWM is demonstrated using the Coral Beowulf cluster at ICASE (for details see reference 9), NASA Langley Research Center.

In this paper the technical details of the RWM are presented first, followed by the presentation of two examples (taken from reference 7) to verify the development. Next a brief introduction to the Coral Beowulf cluster at ICASE, Langley Research Center is provided along with the implementation of the RWM in the cluster. Next Laplace's Equation in a multiply connected domain is analyzed in the Beowulf cluster to measure the speed-up ratio obtained in the multi processor cluster compared to

\footnotetext{
- Aerospace Engineer, Analycical and Computational Methods Branch, Senior Member, AlAA

'Head, Analytical and Computational Methods Branch, Associate Fellow, AlAA

Copyright $\mathbf{O}_{2001}$ by the American Institute of Aeronautics and Astronautics, Inc. No copyright is asserted in the United States under Title 17. U.S. Code. The U.S

Government has a royalty-free license to exercise all rights under the copyright claimed herein for Governmental Purposes. All other rights are reserved by the copyright owner
} 
the single processor implementation. Finally, conclusions from the study are presented.

\section{Technical details of the RWM}

The first step in the development of the RWM for Lapalace's and Poisson's equations for potential flow problems is to define the Itô diffusion processes for Brownian motion based on the mean value theorem. The full details of the method can be found in reference 7 , so only a brief summary presented here. The Brownian motion is a random, zigzag motion of microscopic particles, characterized by the stationary independent increments of non-overlapping time intervals. For example consider a Brownian motion process $\{B(t), t \geq 0\}$ taking place in a time interval, $s<s_{2} \leq s_{3}<t$. The increments $B(t)-B\left(s_{3}\right)$ and $B\left(s_{2}\right)-B(s)$ are independent. For a time interval, $s<t$, the motion of the particle is a Gaussian vector with mean zero and covariance matrix $I(t-s)$, where $l$ denotes the identity matrix. The Itô diffusion processes starts at an arbitrary point $x$ in a domain $\Omega$ at time $t=0$ and defined for a function $g(x)$ in terms of average rate of change of $g(B(t))$ as

$$
E\left[g(B(t)]-g(x)=\frac{1}{2} E\left[\int_{o}^{t} \nabla^{2} g(B(s)) d s\right]\right.
$$

where $\nabla^{2}=\sum_{i=1}^{d} \frac{\partial^{2}}{\partial x_{i}^{2}}$ is the Laplace operator, $E$ is the expectation with the starting point $B(0)=x$, and $d=2$ for two-dimensions and $d=3$ threedimensions.

The Itô formula in Equation (1), can be generalized by replacing $t$ with a random time $T$. The random time $T$ is defined as the time the Brownian motion leaves the domain $D$ for the first time starting at $x \in D$. The averaged Itô formula with $t$ replaced by $T$ can be written as

$$
E\left[g(B(T)]-g(x)=\frac{1}{2} E\left[\int_{o}^{T} \nabla^{2} g(B(s)) d s\right]\right.
$$

This is the key equation for solving the Laplace and Poisson's equations by the RWM.

\section{Laplace's and Poisson's Equations}

Let $u$ be the solution of the Poisson's equation defined in a domain $\Omega$, bounded by a boundary $\Gamma$

$$
\nabla^{2} u(x)+p(x)=0 \quad x \in \Omega
$$

satisfying the Dirichlet boundary conditions

$u(x)=\xi(x), \quad x \in \Gamma_{u}$

where $p$ and $\xi$ are specified functions and $\Gamma$ is the boundary on which the Dirichlet boundary conditions are specified. The value of unknown function $u$ can be written using Itô formula in Equation (2), using $\nabla^{2} u(B(s))=-p(B(s))$ as

$u(x)=E\left[\xi(B(t)]+\frac{1}{2} E\left[\int_{o}^{T} p(B(s)) d s\right]\right.$

The right hand side of equation (5) depends only on the expectations of the known functions $\xi$ and $p$, and samples of the Brownian motion $B$ in the time interval $(0, T)$. The expectation in equation (5) can be estimated using Monte Carlo simulation, since, generally it is not possible to evaluate the integrals analytically. Note that the solution to Laplace's equation can be obtained by substituting $p=0$ in equation (5).

The RWM is most suited for Dirchlet boundary conditions given in equation (4). For mixed boundary conditions the Brownian motion is reflected at the Newmann boundary as described in reference 7 . In the present paper only problems with Dirchlet boundary conditions are considered. 


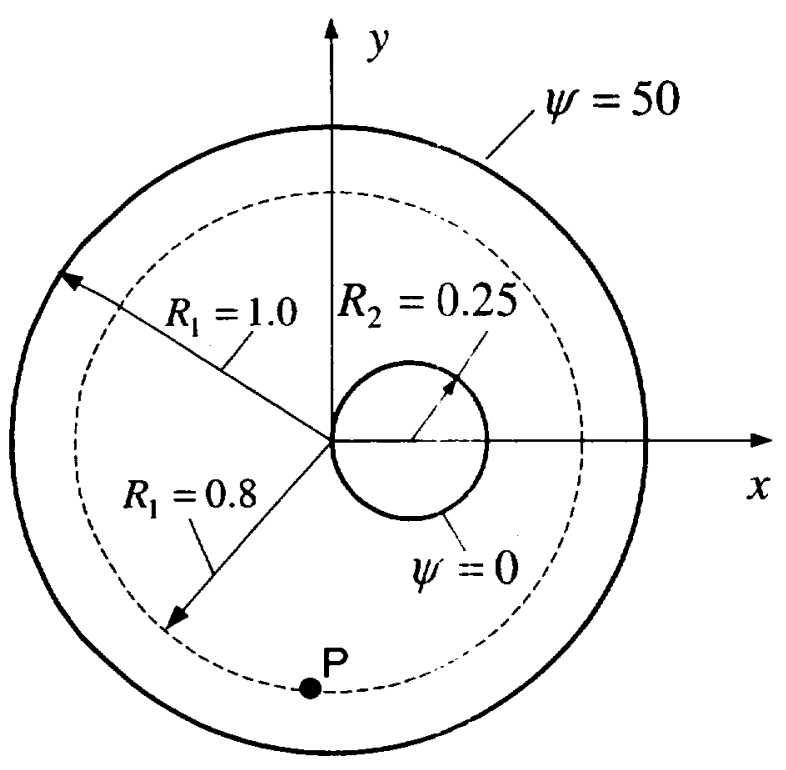

Figure 1. Laplace's equation solved on multiply connected domain

\section{Numerical Examples in a single processor:}

Two examples from reference 5 are selected to verify the RWM in a single processor implementation. These examples are described below:

\section{Laplace's Equation on a Multiply Connected Domain:}

In the first example, Laplace's equation is solved in a multiply connected two-dimensional domain as shown in Figure 1. For the dimensions and boundary conditions shown in Figure 1, the exact solution for the potential at any point within the domain is ${ }^{5}$

$\psi_{\text {exact }}=50\left\{1-\frac{1}{2 \ln \beta} \ln \left[\frac{(x-\alpha)^{2}+y^{2}}{(\alpha x-1)^{2}+\alpha^{2} y^{2}}\right]\right\} ;$

with $\alpha=\beta=2+\sqrt{3}$

The following Monte Carlo simulation procedure was used to calculate the potential along a circle of radius $r$, for example $r=R_{1}=0.8$ (shown as the dotted line in Figure 1).
1. Start the Brownian motion of a particle at any point $P\left(x_{p}, y_{p}\right)$ inside the domain where the solution of the potential $\psi$ is to be determined

2. Select $\left(x_{i}, y_{i}\right)$ as equal to $\left(x_{p}, y_{p}\right)$

3. Select the time step $\Delta t$ for the Brownian motion.

4. The position of the particle at the end of the current step can be determined using the Brownian motion properties as

$$
\begin{aligned}
& x_{i}=x_{i}+\sqrt{\Delta t} \operatorname{Random}(\varepsilon) \\
& y_{i}=y_{i}+\sqrt{\Delta t} \operatorname{Random}(\varepsilon)
\end{aligned}
$$

where $\operatorname{Random}(\varepsilon)$ is a random number generator function that returns a random number from the set of a normally (Gaussian) distributed random numbers with mean zero and unit variance. Note that the incremental steps in the $x$ and $y$ directions are different.

5. Repeat step 4 until the particle reaches either of the two boudaries and exits the domain. Record the value of the potential $\psi_{e}$ at the exit point. (The particle is said to be absorbed at the boundary).

6. Go to step 1 for the next sample in Monte Carlo simulation with the same starting point $\left(x_{p}, y_{p}\right)$

7. Repeat steps 1-5, for $N$ number of samples.

8. Calculate the value of the potential at the point $P\left(x_{P}, y_{P}\right)$ as

$$
\psi_{P}=\frac{1}{N} \sum_{e=1}^{N} \psi_{e}
$$

The time step $(\Delta t)$ and number of samples $N$ are the two important parameters in the RWM. Two sets of parameters $\Delta t=0.01, N=100$ and $\Delta t=0.0001, N=5000$ were selected to study the effect of the parameters time step $\Delta t$ and sample size $N$ on the solution. The potentials are calculated at 21 locations equally spaced around the circle of radius 0.8 . Figure 2 shows the comparison of the potential calculated using the RWM and the exact solution for the two sets of parameters. The solution accuracy 
improves upon decreasing the time step $\Delta t$ and increasing the number of samples $N$.

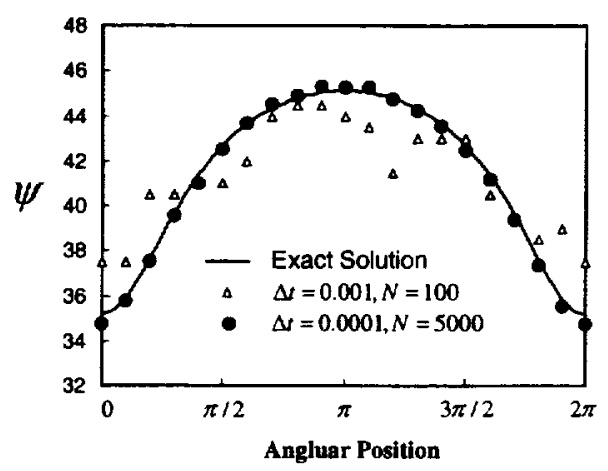

Figure 2. Potential $(\psi)$ along the circle of radius

$$
r=R_{1}=0.8
$$

\section{Poisson's Problem on an ellipsoid:}

The second example is a Possion's problem on a ellipsoid with Dirichlet boundary conditions. The ellipsoid is described by the equation

$$
\frac{x^{2}}{a^{2}}+\frac{y^{2}}{b^{2}}+\frac{z^{2}}{c^{2}}=1
$$

The Dirichlet boundary conditions on the surface of the ellipsoid are applied according to the equation

$\psi_{\text {exact }}=x^{2}+y^{2}+z^{2}$

The ellipsoid major axes are set to unity. $(a=b=c=1.0)$. The potentials along a circle $x^{2}+y^{2}=r^{2}$ (with $r=0.8$ ) on the plane $z=0$ are calculated using the 8 step Monte Carlo simulation procedure described in the previous example. However, the potential at step 5 is calculated using

$$
\psi_{e}=x_{e}^{2}+y_{e}^{2}+z_{e}^{2}
$$

The potentials are calculated at 21 locations along the circumference of the circle. The potentials obtained in the analysis are shown and compared with the reference solution in Figure 3 for $\Delta t=0.1$ and the number samples $N=5000$. Good agreement is shown between the analysis results and the reference solution.

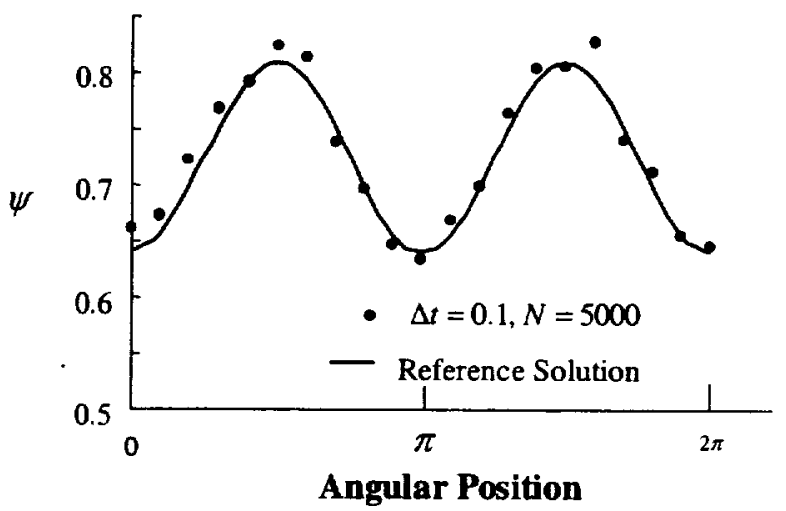

Figure 3. Potential $(\psi)$ along the circle with

$$
x^{2}+y^{2}=(0.8)^{2} \text { and } z=0 \text {. }
$$

\section{Coral Beowulf Cluster at ICASE in Langley Research Center}

Coral is a 96-CPU Beowulf cluster with a dual CPU $400 \mathrm{MHz}$ Pentium II server as the front end, while two dual CPU $500 \mathrm{MHz}$ Pentium III machines act as file servers. The cluster runs with Linux operating sysytem. There are 64 computing nodes consisting of 8 Pentium III single processors at $400 \mathrm{MHz}, 16$ Pentium III dual processors at $500 \mathrm{MHz}, 16$ Pentium III dual processors at $800 \mathrm{MHz}$ and 24 Pentium IV single processors at $1700 \mathrm{MHz}$. The parallel programs on Coral are written using the Message Passing Interface (MPI) standard and are fully described in reference 8 . Three different MPI implementations are available on Coral . While accessing the Coral cluster, the user can request all the computing nodes, can request few selected nodes or can specify the number of nodes required. 


\section{Multiply Connected Domain on Laplace's Eqution in Multi Processor Implementation}

The multiply connected domain shown in Figure 1 of example 1 is used in the multiprocessor environment to calculate the potentials at 21 stations (locations) along the circumference of the circle with radius, $r=R_{1}=0.8$. The number of samples is selected as 12,000 for this case. Two type of multi processor configurations are studied and described below.

Configuration I: Samples shared in processors: In this configuration, for each station, the 12,000 samples are shared across the processors. For example, in an analysis with 4 processors, each processor will handle 3000 samples. The total time taken for the analysis is calculated by summing the time taken for the 21 stations. In other words, in this configuration the stations are sequentially processed, while the number of samples are run in parallel and shared across the cluster.

Configuration II: Stations shared in processors: In this configuration, the 21 stations are run in parallel on 21 processors, while in each processor the 12,000 samples are run sequentially. The total time taken for the analysis is calculated by summing the time taken in each of the 21 processors.

Configurations I and II are run on the Coral Beowulf cluster using $1700 \mathrm{MHzPentium} \mathrm{IV} \mathrm{single} \mathrm{processors}$ nodes. For both the configurations, the processor nodes are divided so that there is one master node and the remaining processors are slave nodes. The master node maintains all the communication to the slave nodes. It also receives the data from slave nodes and compiles it for output.

For Configuration I, the number of processors is varied from 1 to 23 including the master node. For configuration II, 22 processors are used, one for the master node and 21 processors for the 21 stations. The total time taken for the multiprocessor analyses are shown in Figure 4, where it can be seen that the total time for the Configuration I reduced from approximately 16 seconds to 1 second as the number of processors varied from 1 to 23 . The 22 processors in configuration I take almost same the time as the configuration II with 22 processors. This implies that the each random walk is highly independent of the others and little time is wasted in communication between processors.

In order to measure the speed gain in the multiprocessor analysis, a speed -up ratio is defined as

Speed - up ratio $=\frac{\text { Time taken in singleprocessor }}{\text { Time taken in multiple processors }}$

The speed-up ratio is shown in Figure 5 for Configurations I and II. It can be seen that the speed gain of 16 is obtained as the number of processors increased from 1 to 22 .

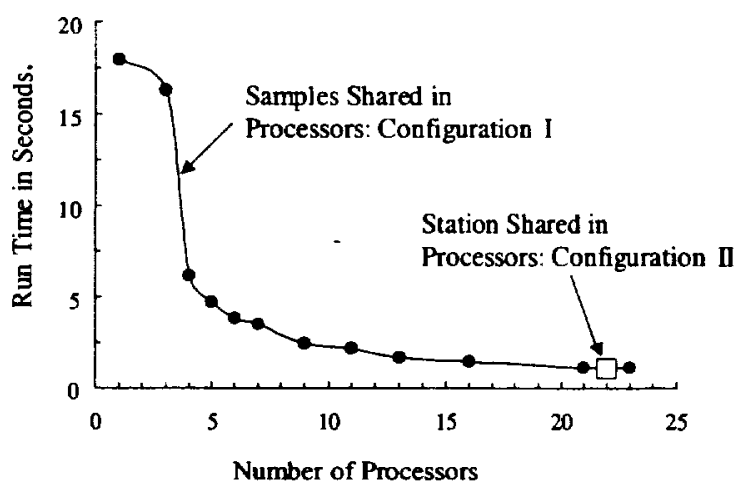

Figure 4 : Variation of analysis time with the number of processors

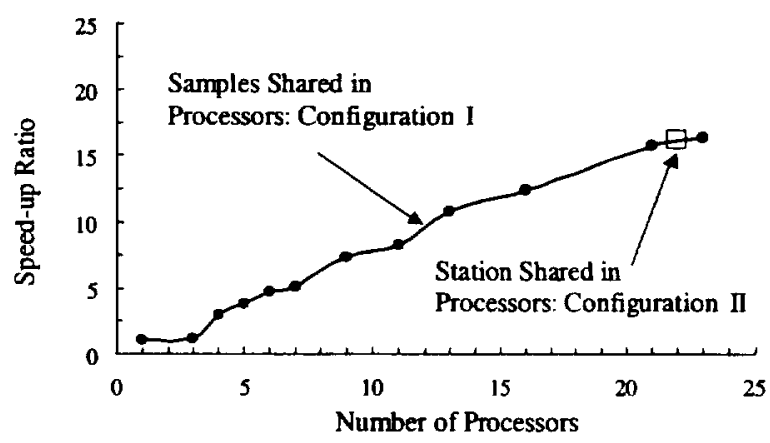

Figure 5. Speed-up ratio in the multi processors analyses 


\section{SUMMARY}

A random walk method for potential problems governed by Lapalace's and Poisson's equations is developed for two- and three- dimensional problems. The method is demonstrated in a Beowulf cluster of computers. A multiply connected domain problem governed by Laplace's equation is analyzed with the number of processors ranging from 1 to 23 . Two types of processors sharing are utilized in the parallel implementation. Using the Multiprocessor parallel method a speed gain of 16 is achieved as the number processors increased from 1 to 22 .

\section{References}

1. Brebbia, C. A., and Dominguez, J., "Boundary Elements: An Introductory Course", Computational Mechanics Publications, McGraw-Hill Book Company, 1992.

2. Raju, I.S., Sistla, R. and Krishnamurthy, T., "A Displacement Gradient BEM for accurate Stress Computation Near Boundaries in 2-D Anisotropic Problems," Computers \& Structures, Vol. 59, pp. 453$462,1996$.

3. Krishnamurthy, T., and Raju, I.S., "Coupling Finite and Boundary Element Methods", International Journal of Numerical Methods in Engineering, Vol. 36, pp. 35953616, 1993

4. .Belytschko, T., Lu, Y.Y., and GU, L., "Element Free Galerkin Methods", International Journal for Numerical Methods in Engineering, Vol. 37, pp. 229 256, 1994.

5. Atluri, S. N., and Zhu, T., "A new Meshless Local Petrov-Galerkin (MLPG) Approach in Computational Mechanics," Computational Mechanics, Vol. 22, pp 117-127, 1998.
6. Raju, I. S. and Chen, T., "Meshless PetrovGalerkin Method Applied to Axisymmetric Problems", AIAA 2001-1253, Proceedings of 42nd AIAA/ASME/ASCE/AHS/ASC Structures, Structural Dynamics, and Materials Conference, Seattle, WA, April 16-19, 2001.

7. Chati, M. K., Girigoriu, M. D., Kulkarni, S.S, and Mukherjee, S., "Random Walk Method for the two- and three-dimensional Laplace, Poisson and Helmholtz's equations", International Journal of Numerical Methods in Engineering, Vol. 51, pp. 1133-1156, 2001.

8. Gropp, William, Lusk, Ewing, and Skjellum, Anthony," Using MPI: Portable Parrallel Programming with the Message-Passing Interface", Second Edition, The MIT Press, Cambridge, Massachusetts, London, England, 1999

9. http://www.icase.edu/Coral 